\title{
High-grade serous carcinoma with discordant p53 signature: report of a case with new insight regarding high-grade serous carcinogenesis
}

Yuichiro Hatano ${ }^{1 *}$ (D, Shinya Fukuda ${ }^{1}$, Hiroshi Makino², Hiroyuki Tomita ${ }^{1}$, Ken-ichirou Morishige ${ }^{2}$ and Akira Hara ${ }^{1}$

\begin{abstract}
Background: Although p53 signature, benign-appearing epithelial cells with p53 diffuse expression, is frequently found in the fallopian tubes, the clinical and pathological significance of this lesion in the case of high-grade serous carcinoma (HGSC) patients still remains unclear.

Case presentation: A 56-year-old woman was referred to the gynecologist on account of abdominal distention. Since radiological and serological workup suggested that her illness was due to advanced ovarian cancer (FIGO Stage IVB), she received neoadjuvant chemotherapy, and the clinical evaluation of the chemotherapeutic response was a partial response. She underwent total hysterectomy with bilateral salpingo-oophorectomy, omentectomy, and intra-pelvic and para-aortic lymphadenectomy. Histologically, the cancer cells showed high-grade nuclear atypia and spread into the bilateral ovaries, omentum, uterine serosa, and left fallopian tube. The cancer cells showed complete absence of p53 but overexpressed p16, whereas some of benign-appearing tubal epithelial cells overexpressed p53 but lacked p16 expression. The results of direct sequence analysis revealed that the ovarian cancer contains a 1 bp deletion in exon 8 of TP53. Finally, the histological diagnosis of HGSC with discordant p53 signature was made. Interestingly, nuclear expression of $\mathrm{Y}-\mathrm{H} 2 \mathrm{AX}$, a well-known marker of DNA damage, was not only observed in both p53 aberrantly-expressing lesions but also the benign-appearing tubal epithelium without p53 overexpression. After the histological confirmation, she received adjuvant chemotherapy and has been in disease-free condition without any detectable tumor for 5 months.

Conclusion: Recent evidence suggests that p53 signature is the putative precursor of p53 overexpression-type HGSC. Because the putative precursors of the other p53 immunophenotypical HGSC are not proposed, we presume Y-H2AX-expressing cells without p53 overexpression may be a potent candidate of null-type TP53-mutated tubal cells, which are named " $Y-H 2 A X$ responsive foci."
\end{abstract}

Keywords: Fallopian tube, Ovary, High-grade serous carcinoma, p53 signature, $\mathrm{Y}-\mathrm{H} 2 \mathrm{AX}$

\section{Background}

Extra-uterine high-grade serous carcinoma (HGSC) is one of the most lethal malignancies of the female genital tract, and almost always harbors TP53 mutation [1-4]. Recent evidence indicates that most HGSC cases arise from serous tubal intraepithelial carcinoma (STIC), which is particularly

\footnotetext{
* Correspondence: yuha@gifu-u.ac.jp

'Department of Tumor Pathology, Gifu University Graduate School of

Medicine, 1-1 Yanagido, Gifu 501-1194, Japan

Full list of author information is available at the end of the article
}

found in the distal fallopian tube $[5,6]$. Concurrent STIC and HGSC likely share a common TP53 mutation and/or p53 expression pattern, indicating that these two serous cancers originate from a single TP53-mutated clone [7, 8]. In addition, no intra-tumoral heterogeneity of TP53 mutation is found in primary untreated HGSC cases [9]. Consistent with this notion, demonstration of identical TP53 mutation is a useful clue of synchronous lesions [10] and late recurrence [11] of HGSC, and vice versa [12]. Therefore, routine pathological diagnosis of HGSC requires

(c) The Author(s). 2018 Open Access This article is distributed under the terms of the Creative Commons Attribution 4.0 International License (http://creativecommons.org/licenses/by/4.0/), which permits unrestricted use, distribution, and 
at least p53 immunostaining and extensive investigation of the fallopian tubes, which is based on the SEE-FIM (Sectioning and Extensively Examining of the Fimbriated end) protocol [13].

Such careful pathological examination of the fallopian tubes incidentally detects the p53 signature, which comprises continuous normal-looking tubal epithelium with p53 overexpression. These aberrant p53-expressing cells are occasionally found in asymptomatic healthy women regardless of $B R C A$ germline mutation status, and more frequently in women with tubal intraepithelial carcinoma [14]. Interestingly, some of these benignappearing lesions possess TP53 mutation identical to the coexisting tubal intraepithelial carcinoma. Considering that p53 dysregulation is believed to be the initiating event for high-grade serous carcinogenesis, the p53 signature is a potent precursor of STIC and/or HGSC.

We herein report the case of a patient with two distinctive p53 aberrantly-expressing lesions that suggest some new insight into the understanding of high-grade serous carcinogenesis.

\section{Case presentation}

\section{Clinical history}

A 56-year-old woman, gravida 2, para 2, was referred to the gynecologist on account of abdominal distention. She had a past medical history of acute pancreatitis, but she had never experienced similar symptoms before. She was on no medications at the time of presentation. She denied familial history of ovarian and/or breast cancer. Blood tests revealed that serum CA125 was high $(1520.5 \mathrm{U} / \mathrm{mL})$. Abdominopelvic magnetic resonance imaging showed massive ascites, ovarian masses and numerous nodules in the abdominal and pelvic cavities. In addition, chest computed tomography (CT) scan showed left-supraclavicular lymphadenopathy. Consistent with these radiological findings, positron emission tomography-CT detected fluorodeoxyglucose accumulation within bilateral ovarian masses and left-supraclavicular, peritoneal, para-aortic and intra-pelvic nodules, which were suggestive of distant metastases and peritoneal dissemination from ovarian cancer. Since the clinical diagnosis of advanced ovarian cancer, FIGO Stage IVB (cT3N1M1) was made, three cycles of

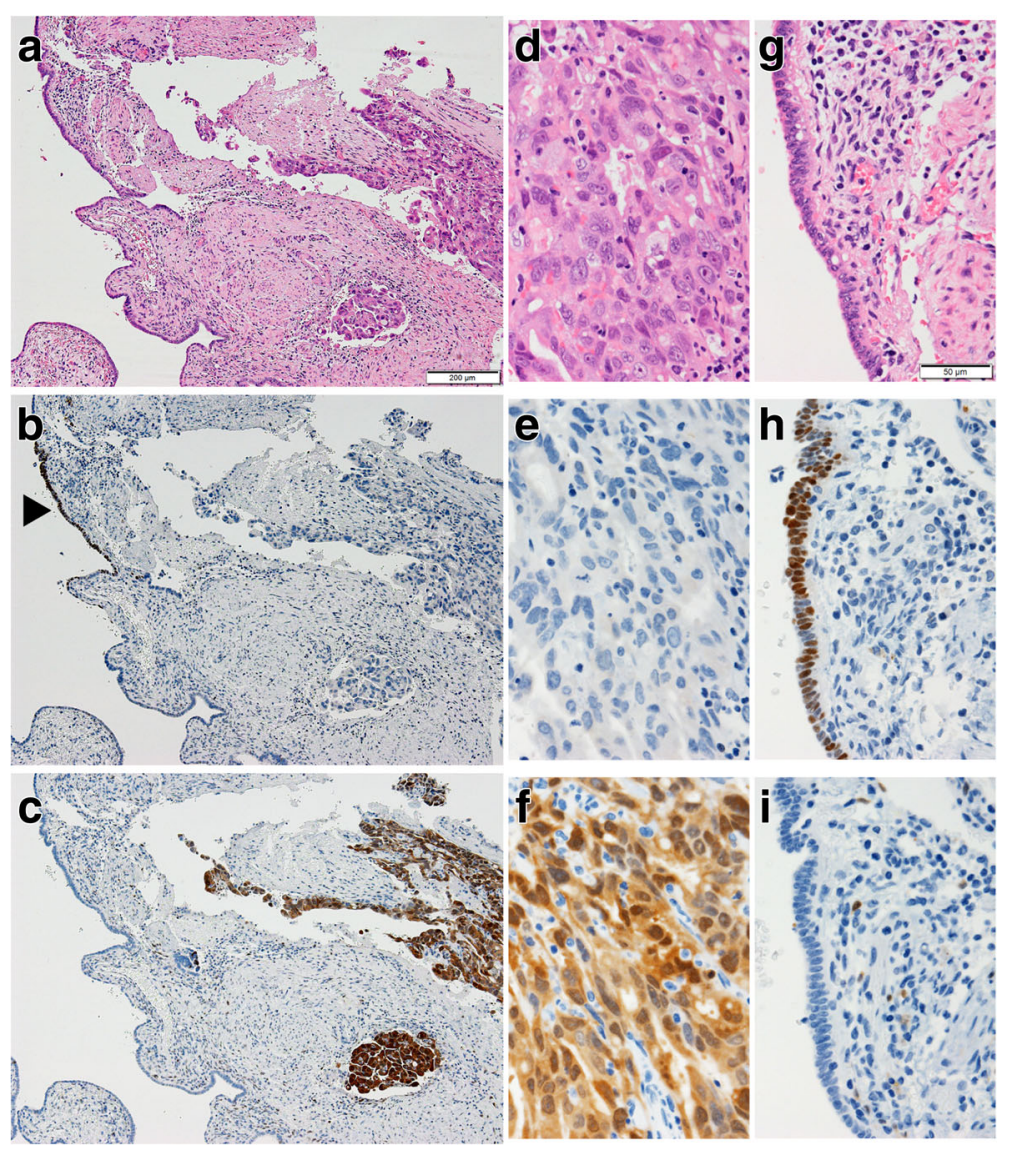

Fig. 1 Two distinctive p53 aberrantly-expressing lesions. (a)-(c) Representative hematoxylin and eosin stained (a), p53 (b) and p16 (c) immunostained images of the left fallopian tube, which contains high-grade serous carcinoma (right) and p53 signature (left, arrowhead). (d)-(f) Representative hematoxylin and eosin stained (d), p53 (e) and p16 (f) immunostained images of high-grade serous carcinoma. (g)-(i) Representative hematoxylin and eosin stained (g), p53 (h) and p16 (i) immunostained images of p53 signature 
neoadjuvant chemotherapy (NAC) with paclitaxel/carboplatin were administered. Following NAC, serum CA125 was reduced $(97.6 \mathrm{U} / \mathrm{mL})$ on blood tests, all the suspicious tumoral lesions had decreased in size, and the ascites had diminished on radiological re-assessment. The clinical evaluation of chemotherapeutic response yielded a partial response; total hysterectomy with bilateral salpingooophorectomy, omentectomy, intra-pelvic and para-aortic lymphadenectomy were performed.

Upon histological assessment of the surgical specimen the ovarian cancer was classified as FIGO Stage IIIB (ypT3bN1MX) [15] and the chemotherapy response score was estimated as 1 (minimal tumor response) [16]. She underwent six cycles of adjuvant chemotherapy with bevacizumab and/or paclitaxel/carboplatin, and has been disease-free without any detectable tumor for 5 months.

\section{Pathological findings}

Histologically, the cancer cells showed high-grade nuclear atypia and spread into both ovaries, the omentum, uterine serosa, and left fallopian tube. In the left distal fallopian tube (Fig. 1a-c), these cancer cells (Fig. 1d) showed complete absence of p53 (clone: DO-7; Figs. 1e and 2a), but overexpressed p16 (Figs. 1f and 2b). Interestingly, the benign-appearing tubal epithelium adjacent to the highgrade cancer cells (Fig. 1g) showed an overexpression of p53 (Figs. 1h and 2a), but lacked p16 expression (Figs. 1i and $2 \mathrm{~b}$ ). In addition, nuclear expression of $\gamma-\mathrm{H} 2 \mathrm{AX}$ (clone: ab11174, Abcam, Fig. 2c), was found in both p53 aberrantly-expressing lesions. The Ki-67 labeling index of p53-positive benign-appearing epithelial cells was $<5 \%$ (Fig. 2d), whereas that of high grade tumor cells was approximately 15\% (Fig. 2e). Finally, we diagnosed these lesions as HGSC with discordant p53 signature.

We also performed TP53 mutation analysis of HGSC by direct sequencing according to the International Agency for Research on Cancer protocol (http://p53.iarc.fr/Download/TP53_DirectSequencing_IARC.pdf Accessed 22 Dec 2017) with minor modification. Briefly, genomic DNA was extracted from formalin-fixed, paraffin-embedded samples of the ovarian tumor and normal tissue, respectively, using NucleoSpin DNA FFPE XS (Macherey-Nagel, Germany). Exons 4-8 of TP53 were amplified by polymerase chain reaction and the products were analyzed at the Division of Genomics Research, Life Science Research Center, Gifu University. The results showed that the ovarian cancer contains a 1 bp deletion (c.792del1), which is interpreted as a frameshift mutation, in exon 8 of TP53, whereas normal tissue lacked this alteration (Fig. 3).

\section{Discussion}

The patterns of p53 aberrant expression, which is a high-precision predictor of TP53 mutation, have been traditionally divided into diffuse positive (also known as

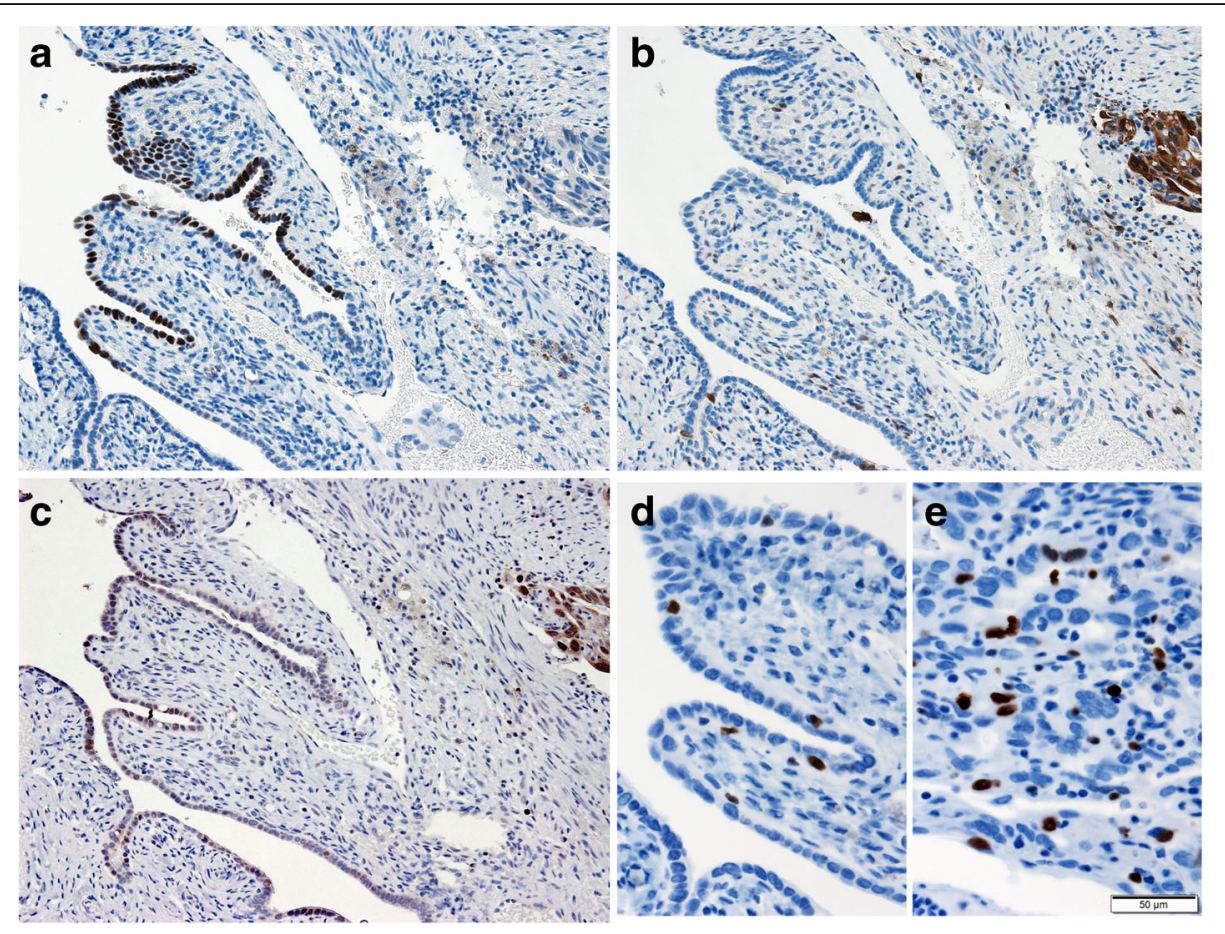

Fig. 2 Y-H2AX expression in the distal fallopian tube. (a)-(c) Representative immunostained images of the p53 (a), p16 (b) and $\gamma-\mathrm{H} 2 \mathrm{AX}(\mathbf{c})$ protein expressions in the high-grade serous carcinoma (right) and p53 signature (left). (d)(e) Representative Ki-67 immunostained images of p53 signature (d) and high-grade serous carcinoma (e) 


\section{G T G G TA A T T T T C TGGGAC}

10
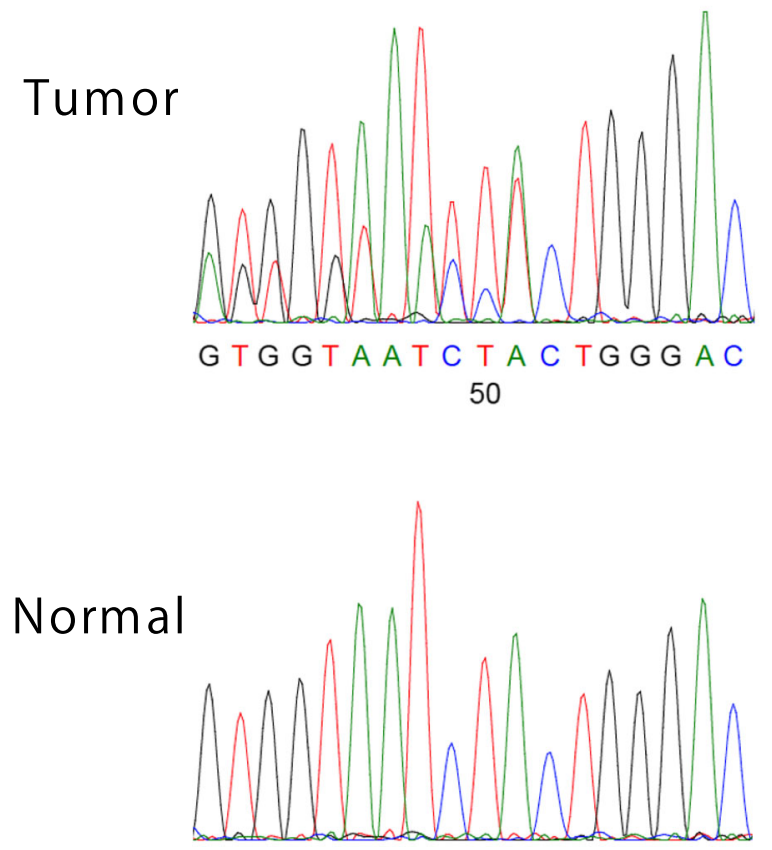

Fig. 3 TP53 mutation analysis of HGSC. A DNA sequence analysis of TP53 exon 8 from the ovarian and normal tissue with the reverse primer. The sequence of the ovarian tumor shows biallelic pattern from the coding DNA reference number 792, indicating that HGSC harbors a 1 bp deletion (c.792del1) in the TP53 gene

overexpression), and complete absence (also called as null, diffuse negative) [9]. Intriguingly, the pattern of p53 aberrant expression also predicts the TP53 mutation status. The overexpression and null patterns are mainly associated with gain-of-function and loss-of-function type TP53 mutations, respectively. Recently, Kobel et al. reported the cytoplasmic p53 staining, which is the rare third pattern of p53 aberrant expression that arises from genetic mutation in the nuclear localization signaling domain [3]. Collectively, abnormal patterns of p53 expression reflect TP53 mutation types in HGSC.
In this case, two distinctive p53 aberrantly-expressing lesions were confirmed in the fallopian tube section. Direct sequencing revealed that HGSC harbored a $1 \mathrm{bp}$ deletion in exon 8 of TP53. This alteration leads to disruption of the p53 DNA binding domain, which is consistent with the complete absence of p53 expression in HGSC. On the other hand, we failed to investigate TP53 mutation status of p53 signature because of the insufficient quantity. Given that TP53 mutation is an initial and central factor in the pathogenesis of HGSC, different TP53 mutation types likely determine the biological properties of neoplastic cells, including the p53 expression pattern. Thus, discordant p53 aberrantly-expressing patterns are interpreted as the p53 signature being unrelated to the coincident p53 null-type HGSC.

The various p53 immunophenotypes in HGSC implicate the existence of corresponding p53 immunophenotypical precursors. In other words, benign-looking tubal epithelium with abnormal p53 expression includes not only conventional overexpression-type p53 signature but also null- or cytoplasmic staining-type p53 signature (Table 1). Although the conventional p53 signature is detected by routine diagnostic immunohistochemistry, cytoplasmic staining-type p53 signature is probably misdiagnosed as the overexpression-type because of the rare and unknown abnormal pattern. Besides, the other speculative "nulltype" p53 signature must be undetectable in the lesion by the same immunohistochemical analysis. Conceptually, these p53 null-type normal-looking tubal epithelial cells should be distinguished from secretory cell outgrowth (SCOUT) [17], which lacks TP53 mutation.

To demonstrate invisible p53 mutants, we performed additional immunohistochemical analysis of $\gamma$-H2AX, a well-known marker of DNA damage [18, 19]. Since the orthodox function of wild-type p53 is activation of the DNA repair system, foci of $\gamma-\mathrm{H} 2 \mathrm{AX}$-expressing cells may reflect p53 dysfunction and/or TP53 mutations in the cells. Consistent with a previous report, [14] both the p53 signature and HGSC expressed $\gamma-\mathrm{H} 2 \mathrm{AX}$. Noteworthy, nuclear expression of $\gamma-\mathrm{H} 2 \mathrm{AX}$ was also detected in the benign-appearing tubal epithelium without p53 overexpression (Fig. 2c). Similarly, Staff et al. reported that $\gamma$ H2AX-expressing cells were often found in the fallopian

Table 1 Summary of relationship between high-grade serous carcinogenesis and p53 immunophenotype

\begin{tabular}{lll}
\hline p53 immunophenotype & Precursor lesion & Malignant form \\
\hline $\begin{array}{l}\text { Diffuse positive } \\
\text { (also called overexpression) }\end{array}$ & p53 signature [14] & HGSC with p53 overexpression, which may reflect GOF p53 \\
$\begin{array}{l}\text { Diffuse negative } \\
\text { (also called as null, complete absence) }\end{array}$ & $\begin{array}{l}\text { Y-H2AX responsive foci, } \\
\text { see Discussion section }\end{array}$ & HGSC without p53 expression, which may reflect LOF p53 \\
Cytoplasmic staining & $\begin{array}{l}\text { Not proposed, but probably } \\
\text { misinterpreted as a p53 signature }\end{array}$ & $\begin{array}{l}\text { HGSC with p53 cytoplasmic expression, which is a rare immunophenotype } \\
\text { wild-type }\end{array}$ \\
SCOUT [17] & HGSC NOS, which shows any p53 immunophenotype
\end{tabular}

HGSC high-grade serous carcinoma, GOF gain-of-function, LOF loss-of-function, SCOUT secretory cell outgrowth, NOS not otherwise specified 
tube but that not all cells overlapped with p53 expression [20]. We speculate that these $\gamma$-H2AX-expressing cells without p53 overexpression may be potent candidates for null-type TP53 mutations, and therefore, named them " $\gamma$ $\mathrm{H} 2 \mathrm{AX}$ responsive foci." Interestingly, the Ki-67 labeling index of these cells was almost the same as that of p53 signature. However, it is very difficult to determine whether these candidate cells harbor TP53 mutation by immunohistochemistry alone, unlike the p53 signature. To identify the hidden precursors of null-type p53 HGSC, other efficient markers of loss-of-function p53 and TP53 mutation analysis are desired. Taken together, the detection of null-type TP53 mutation in benign-appearing tubal epithelial cells can help elucidate unknown precursors of STIC and/or HGSC and certainly improve our understanding of microscopic TP53-mutated intraepithelial lesions.

Molecular characteristics of HGSC are classified as inevitable TP53 mutations and diverse gene expression profiles. TP53-mutated cells variously evolve by genomic instability, and result in intra- and inter-tumoral heterogeneity in HGSC. These heterogeneities often become a problem in medical practice today [21]. To understand the heterogeneities of prognoses and therapeutic response, molecular classification of HGSC based on gene expression profiles has been attempted [2, 22, 23]. Similarly, p53 expression/TP53 mutation type in HGSC has shown promise in the classification of the biological characteristics of this lethal high-grade malignancy.

\section{Conclusions}

Recent evidence suggests the precise prediction of TP53 mutation status solely by p53 immunohistochemistry. This suggestion is also a modest clue to investigate the microscopic lesion, p53 signature. If p53 dysfunction is visualized by other immunohistochemical markers, speculative "null-type" p53 signature could be detected. Therefore, we propose " $\gamma-\mathrm{H} 2 \mathrm{AX}$ responsive foci" as a hidden precursor of null-type p53 HGSC. To progress in the study of the initial genetic event in high-grade serous carcinogenesis, identification of the null- and/or cytoplasmic staining-type TP53-mutated normally-appearing cells is required in the future.

\section{Abbreviations \\ CT: Computed tomography; FIGO: The International Federation of Gynecology and Obstetrics; HGSC: High-grade serous carcinoma; IARC: International Agency for Research on Cancer; NAC: Neoadjuvant chemotherapy; SCOUT: Secretory cell outgrowth; STIC: Serous tubal intraepithelial carcinoma}

\section{Funding}

This work was supported by JSPS KAKENHI (18K15207)

\section{Availability of data and materials}

The datasets used and/or analyzed during the current study are available from the corresponding author upon reasonable request.

\section{Authors' contributions}

YH: conception, pathological diagnosis, and writing of manuscript. YH, SF: TP53 mutation analysis. HM: collection of clinical data. YH, HT: immunohistochemical analysis. KM, AH: revision of manuscript. All authors read and approved the final manuscript prior to submission.

Ethics approval and consent to participate

Not applicable.

\section{Consent for publication}

Written informed consent was obtained from the patient for the publication of this case report. A copy of the consent form is available for review by the Editor of this journal.

\section{Competing interests}

The authors declare that they have no competing interests.

\section{Publisher's Note}

Springer Nature remains neutral with regard to jurisdictional claims in published maps and institutional affiliations.

\section{Author details}

'Department of Tumor Pathology, Gifu University Graduate School of Medicine, 1-1 Yanagido, Gifu 501-1194, Japan. ${ }^{2}$ Department of Obstetrics and Gynecology, Gifu University Graduate School of Medicine, Gifu 501-1194, Japan.

Received: 9 February 2018 Accepted: 18 April 2018

Published online: 27 April 2018

\section{References}

1. Ahmed AA, Etemadmoghadam D, Temple J, Lynch AG, Riad M, Sharma R, et al. Driver mutations in TP53 are ubiquitous in high grade serous carcinoma of the ovary. J Pathol. 2010;221:49-56.

2. Cancer Genome Atlas Research Network. Integrated genomic analyses of ovarian carcinoma. Nature. 2011;474:609-15.

3. Kobel M, Piskorz AM, Lee S, Lui S, LePage C, Marass F, et al. Optimized p53 immunohistochemistry is an accurate predictor of TP53 mutation in ovarian carcinoma. J Pathol Clin Res. 2016;2:247-58.

4. Cole AJ, Dwight T, Gill AJ, Dickson KA, Zhu Y, Clarkson A, et al. Assessing mutant p53 in primary high-grade serous ovarian cancer using immunohistochemistry and massively parallel sequencing. Sci Rep. 2016:6:26191.

5. Singh N, McCluggage WG, Gilks CB. High-grade serous carcinoma of tuboovarian origin: recent developments. Histopathology. 2017;71:339-56.

6. Meserve EEK, Brouwer J, Crum CP. Serous tubal intraepithelial neoplasia: the concept and its application. Mod Pathol. 2017:30:710-21.

7. Kuhn E, Kurman RJ, Vang R, Sehdev AS, Han G, Soslow R, et al. TP53 mutations in serous tubal intraepithelial carcinoma and concurrent pelvic high-grade serous carcinoma-evidence supporting the clonal relationship of the two lesions. J Pathol. 2012;226:421-6.

8. McDaniel AS, Stall JN, Hovelson DH, Cani AK, Liu CJ, Tomlins SA, et al. Nextgeneration sequencing of tubal intraepithelial carcinomas. JAMA Oncol. 2015;1:1128-32.

9. Yemelyanova A, Vang R, Kshirsagar M, Lu D, Marks MA, Shih le M, et al. Immunohistochemical staining patterns of p53 can serve as a surrogate marker for TP53 mutations in ovarian carcinoma: an immunohistochemical and nucleotide sequencing analysis. Mod Pathol. 2011;24:1248-53.

10. Jia L, Yuan Z, Wang Y, Cragun JM, Kong B, Zheng W. Primary sources of pelvic serous cancer in patients with endometrial intraepithelial carcinoma. Mod Pathol. 2015;28:118-27.

11. Anglesio MS, O'Neill CJ, Senz J, Gilks CB, McCluggage WG. Identical TP53 mutations provide evidence that late recurring tubo-ovarian high grade serous carcinomas do not represent new peritoneal primaries. Histopathology. 2017;71:1014-7.

12. Furuya M, Sato T, Tanaka R, Yamamoto M, Yokota NR, Miyagi E. Metachronous serous endometrial intraepithelial carcinoma and serous peritoneal carcinoma: analysis of probable independent lesions. Diagn Pathol. 2016;11:130. 
13. Medeiros F, Muto MG, Lee Y, Elvin JA, Callahan MJ, Feltmate C, et al. The tubal fimbria is a preferred site for early adenocarcinoma in women with familial ovarian cancer syndrome. Am J Surg Pathol. 2006;30:230-6.

14. Lee Y, Miron A, Drapkin R, Nucci MR, Medeiros F, Saleemuddin A, et al. A candidate precursor to serous carcinoma that originates in the distal fallopian tube. J Pathol. 2007;211:26-35.

15. Prat J. FIGO committee on gynecologic oncology. Staging classification for cancer of the ovary, fallopian tube, and peritoneum. Int J Gynaecol Obstet. 2014;124:1-5.

16. Böhm S, Faruqi A, Said I, Lockley M, Brockbank E, Jeyarajah A, et al. Chemotherapy response score: development and validation of a system to quantify histopathologic response to neoadjuvant chemotherapy in Tuboovarian high-grade serous carcinoma. J Clin Oncol. 2015;33:2457-63.

17. Chen EY, Mehra K, Mehrad M, Ning G, Miron A, Mutter GL, et al. Secretory cell outgrowth, PAX2 and serous carcinogenesis in the fallopian tube. J Pathol. 2010;222:110-6.

18. Lukas J, Lukas C, Bartek J. More than just a focus: the chromatin response to DNA damage and its role in genome integrity maintenance. Nat Cell Biol. 2011;13:1161-9.

19. Siddiqui MS, Francois M, Fenech MF, Leifert WR. Persistent gammaH2AX: a promising molecular marker of DNA damage and aging. Mutat Res Rev Mutat Res. 2015;766:1-19.

20. Staff S, Tolonen T, Laasanen SL, Mecklin JP, Isola J, Mäenpää J. Quantitative analysis of gamma-H2AX and p53 nuclear expression levels in ovarian and fallopian tube epithelium from risk-reducing salpingo-oophorectomies in BRCA1 and BRCA2 mutation carriers. Int J Gynecol Pathol. 2014;33:309-16.

21. Zhang M, Zhuang G, Sun X, Shen Y, Wang W, Li Q, et al. TP53 mutationmediated genomic instability induces the evolution of chemoresistance and recurrence in epithelial ovarian cancer. Diagn Pathol. 2017;12:16.

22. Verhaak RG, Tamayo P, Yang JY, Hubbard D, Zhang H, Creighton CJ, et al. Prognostically relevant gene signatures of high-grade serous ovarian carcinoma. J Clin Invest. 2013;123:517-25.

23. Konecny GE, Wang C, Hamidi H, Winterhoff B, Kalli KR, Dering J, et al. Prognostic and therapeutic relevance of molecular subtypes in high-grade serous ovarian cancer. J Natl Cancer Inst. 2014;106:dju249.

\section{Ready to submit your research? Choose BMC and benefit from:}

- fast, convenient online submission

- thorough peer review by experienced researchers in your field

- rapid publication on acceptance

- support for research data, including large and complex data types

- gold Open Access which fosters wider collaboration and increased citations - maximum visibility for your research: over $100 \mathrm{M}$ website views per year

At BMC, research is always in progress.

Learn more biomedcentral.com/submissions 